\title{
Chemical biology and structural studies on the mechanism of regulation of phosphoinositide- dependent protein kinase 1 (PDK1)
}

\author{
LZF. Gross ${ }^{1}$, M. Sacerdoti ${ }^{1}$, AE. Leroux ${ }^{1}$, A. Ghode ${ }^{4}$, GS. Anand ${ }^{4}$, JO. Schulze ${ }^{2}$, MA. Graewert ${ }^{5}$, DI. Svergun ${ }^{5}$, \\ S. Klinke ${ }^{3} \&$ RM. Biondi ${ }^{1,2}$
}

${ }^{1}$ Instituto de Investigación en Biomedicina de Buenos Aires (IBioBA) - CONICET - Partner Institute of the Max Planck Society, Argentina; ${ }^{2}$ Department of Internal Medicine I, Universitätsklinikum Frankfurt, Germany; ${ }^{3}$ Fundación Instituto Leloir, IIBBACONICET, Argentina; ${ }^{4}$ Department of Biological Sciences, National University of Singapore, Singapore; ${ }^{5}$ European Molecular Biology Laboratory (EMBL), DESY Hamburg, Germany

lgross@ibioba-mpsp-conicet.gov.ar

Phosphoinositide-dependent protein kinase 1 (PDK1) is a master AGC kinase of the PI3K signalling pathway that phosphorylates at least other 23 AGC kinases, being PKB/Akt the most relevant substrate for growth and cell survival, and therefore a potential drug target for cancer treatment. Over the years, our laboratory used a chemical and structural biology approach to study and characterize in detail the allosteric regulation of the catalytic domain of PDK1. We developed small compounds that bind to a regulatory site we termed the PIF-pocket and activate PDK1, mimicking the mechanism of activation of AGC kinases by phosphorylation. Using an integrative approach between biochemistry, crystallography and molecular dynamics, we showed how PS653, a small compound that binds to the active ATP-Binding site, displaces through a reverse allosteric mechanism the in vitro interaction between the PIF-pocket and PIFtide, which is a peptide derived from the hydrophobic motif of a PDK1 substrate. Thus, we not only demonstrated an allosteric regulation from a regulatory site to the active site, but also showed experimentally the existence of the reverse process [1]. This bidirectional allosteric mechanism of regulation between both pockets can therefore be modulated by small molecules that bind to their specific orthosteric site and either enhance or inhibit interactions at the allosteric site. Taking this into consideration, it is not surprising that while the pharmaceutical industry has been developing compounds that bind at the ATP-binding site of kinases, they unwillingly developed drugs that affect protein-protein interactions [2]. Moreover, we now provide further evidence of the bidirectional system using hydrogen/deuterium exchange (HDX) experiments and present a rather complete model for a kinase that can be modulated bidirectionally with small compounds. This concept of bidirectional allostery in kinases can be exploited to produce drugs that enhance or disrupt the formation of multi-protein complexes. Could this mechanism be already in use physiologically? We found out that adenosine binds at the ATP-binding site and allosterically enhances the interaction between PIFtide and PDK1, which demonstrates that bidirectional allostery is a phenomenum that can also be modulated by metabolites. But interestingly, adenine, AMP, ADP, or ATP do not produce this effect. The findings open the possibility that the physiological regulation of the kinase complexes may be modulated by metabolites and implies that the metabolic state of cells could be linked to cell signalling.

As a master kinase tightly regulated, PDK1 possesses a selective activation of substrates such as SGK or S6K, which in order to be phosphorylated require a docking interaction of their C-terminal hydrophobic motifs with the PIF-Pocket of PDK1. However, this is not the case of Akt/PKB, since it can be activated in a PIF-Pocket independent way. In this line, we and others showed that small compounds that bind to the PIFpocket of PDK1 block the phosphorylation of S6K, but do not affect the phosphorylation of PKB/Akt by PDK1[3]. However, up to date little is known about the mechanistic and structural details of PDK1 full length. We are currently using an interdisciplinary approach to understand how the full-length protein is regulated and if this regulation mechanism can be manipulated to specifically inhibit the activation of PKB/Akt. As a result of a medium-scale screening of small compounds, we validated a series of "hits" that modulate PDK1 structure by interaction at different sites on PDK1. We here present a series of results obtained using HDX and SAXS experiments on full length PDK1, as well as the crystal structure of the catalytic domain of PDK1 bound to a small compound that stabilizes a particular PDK1 conformation. The new data is used to present an updated model on the molecular mechanism of regulation of full length PDK1, in which we not only show the existence of bidirectional allostery but also the existence of 3 different conformations of full length PDK1.

[1] Schulze, J.O., Saladino, G., Busschots, K., Neimanis, S., Suess, E., Odadzic, D., Zeuzem, S., Hindie, V., Herbrand, A.K., Lisa, M.N., Alzari, P.M., Gervasio, F.L. and Biondi, R.M. Bidirectional Allosteric Communication between the ATP-Binding Site and the Regulatory PIF Pocket in PDK1 Protein Kinase. Cell Chem Biol, 2016. 23(10): p. 1193-1205.

[2] Leroux, A.E. and Biondi R.M, Renaissance of Allostery to Disrupt Protein Kinase Interactions. Trends Biochem Sci, 2020.

[3] Busschots, K., Lopez-Garcia, L.A., Lammi, C., Stroba, A., Zeuzem, S., Piiper, A., Alzari, P.M., Neimanis, S., Arencibia, J.M., Engel, M., Schulze, J.O. and Biondi, R.M., Substrate-Selective Inhibition of Protein Kinase PDK1 by Small Compounds that Bind to the PIF-Pocket Allosteric Docking Site. Chem Biol, 2012. 19(9): p. 1152-63

Keywords: Allostery; Regulation; Kinases; Metabolites; Small Compounds

Acta Cryst. (2021), A77, C1114 